\title{
AVALIAÇÃO DA MORFOLOGIA APICAL DE INCISIVOS INFERIORES EM DIFERENTES NÍVEIS
}

EVALUATION OF THE MORPHOLOGY OF APICAL LOWER INCISORS AT DIFFERENT LEVELS

\author{
Marilia Fagury Videira Marceliano-Alves \\ Eduardo Fagury Videira Marceliano \\ Patrícia de Almeida Rodrigues Silva e Souza
}

\author{
Doutoranda em Endodontia pela Universidade \\ do Estado do Rio de Janeiro \\ Especialista em Prótese Dentária pela Odontoclínica \\ Central do Exército. \\ Doutora em Endodontia pela Faculdade \\ de Odontologia de Bauru
}

\section{Resumo}

Com objetivo de avaliar a morfologia do canal, foram coletados 100 incisivos inferiores com apicetogênese completa. Os dentes foram seccionados com disco de aço dupla-face em diferentes níveis apicais $(1,3$, e $5 \mathrm{~mm})$, e para avaliação foram dispostos sobre lâmina de vidro para histologia. Os cortes foram levados à lupa estéreo-microscópica para análise da morfologia do canal. Os resultados demonstraram que o formato oval longo foi encontrado em $48 \%$ dos canais a $1 \mathrm{~mm} ; 64 \%$ a $3 \mathrm{~mm}$ e $5 \mathrm{~mm}$. O formato oval foi encontrado em $36 \%$ dos canais estudados a $1 \mathrm{~mm}$ e $5 \mathrm{~mm}$ do ápice e o formato circular foi encontrado apenas a $1 \mathrm{~mm}$ do ápice (16\%). A frequência de dois canais é baixa, perfazendo um total de $8 \%$. Os resultados demonstraram que a incidência de canais ovais longos é grande no terço apical, e, portanto, os preparos circulares promovidos pela instrumentação podem não atingir todas as paredes do canal radicular, deixando áreas intocadas com restos de material infectado, ficando a limpeza a cargo das substâncias químicas auxiliares, o que pode levar ao insucesso da terapia.

Palavras-chave: morfologia do canal RADICULAR - CANAIS OVAIS - SECÇÃO TRANSVERSAL.

\section{Abstract}

In order to evaluate the canal's morphology, we collected 100 lower incisors with complete apexification. The teeth were cut with double-sided steel disk at different apical levels (1,3 and $5 \mathrm{~mm}$ ), and they were placed on a glass slide for histological evaluation. The cuts were taken to the stereo-microscope for a canal morphology analysis. Results showed that the long-oval format was found in $48 \%$ of the canals at $1 \mathrm{~mm}, 64 \%$ at $3 \mathrm{~mm}$ and $5 \mathrm{~mm}$. The oval format was found in $36 \%$ of the studied canals at $1 \mathrm{~mm}$ and $5 \mathrm{~mm}$ from the apex; the circular form was found at only $1 \mathrm{~mm}$ from the apex (16\%). The frequency of two canals is low, making a total of $8 \%$. Results showed that the incidence of long oval canals is large in the third apical, and so the circular preparations promoted by the instrumentation may not reach all the walls of the root canal leaving untouched areas with remains of infected material, and leaving the cleansing to the auxiliary chemicals, which can lead to the therapy's failure.

KEYWORDS: ROOT CANAL MORPHOLOGY - OVAL CANALS - CROSS SECTION. 


\section{INTRODUÇÃO}

Ao se analisar a morfologia dos incisivos inferiores, a literatura relata um achatamento acentuado no sentido mésio-distal, o que pode gerar um segundo canal localizado lingualmente ao principal, bem como a ocorrência de canais severamente achatados, de seções transversais alongadas, sendo o maior diâmetro no sentido vestíbulo lingual. ${ }^{1}$

Em virtude desta diversidade anatômica encontrada em canais de incisivos inferiores, podem permanecer áreas não abrangidas pelo preparo, como canais linguais não detectados, e ainda em canais achatados, paredes não tocadas pelas limas, deixando restos pulpares e dentina infectada em áreas localizadas vestíbulolingualmente. ${ }^{2}$ Tal fato pode comprometer a terapia, pois deixa a limpeza somente a cargo das substâncias químicas auxiliares, e ainda dificulta o acesso da medicação intracanal à intimidade dos túbulos dentinários. ${ }^{3}$

Em estudo avaliando a morfologia de 300 dentes anteriores mandibulares clarificados, Vertucci $^{4}$ descreveu $30 \%$ de incisivos centrais e $25 \%$ de incisivos centrais com dois canais. Em estudo semelhante, Miyashita et al. ${ }^{5}$ avaliaram 1.085 incisivos inferiores diafanizados, reportaram $12,4 \%$ de dentes com dois canais e cerca de $3 \%$ apresentaram mais de uma foramina apical.

Por sua vez, Mauger et al., ${ }^{2}$ que avaliaram a morfologia de 100 incisivos inferiores em diferentes níveis transversais, relataram a presença de dois canais em $30 \%$ dos incisivos centrais e em $25 \%$ dos incisivos laterais avaliados a $1 \mathrm{~mm}$ e $3 \mathrm{~mm}$ aquém do ápice radicular, e ainda relataram a presença de canais achatados em $20 \%$ a $1 \mathrm{~mm}, 30 \%$ a $2 \mathrm{~mm}$, e $55 \%$ a $3 \mathrm{~mm}$ aquém do ápice radicular.

Estes dados foram semelhantes aos encontrados por Al-Quadah e Awawdeh, ${ }^{6}$ que por meio da diafanização identificaram o segundo canal em 26,2\% dos incisivos avaliados, e ainda descreveram que apenas $8,7 \%$ apresentaram duas foraminas apicais, sendo considerado um achado raro.

Avaliando a incidência de canais achatados em 180 dentes de diferentes grupos, em diferentes secções apicais, Wu et al. ${ }^{3}$ relataram que em incisivos inferiores foram observados $10 \%$ de canais achatados a $1 \mathrm{~mm}, 55 \%$ a $2 \mathrm{~mm}$, e $40 \%$ a $3 \mathrm{~mm}$ do ápice radicular.

Baseados no estudo de Wu et al. ${ }^{3}$, Uma et al. ${ }^{7}$ avaliaram a seção transversal de 50 incisivos inferiores, relatando que cerca de $16 \%$ dos dentes avaliados apresentaram canais achatados nos níveis apicais estudados (1, 2, $3 \mathrm{~mm})$.

A literatura é unânime em considerar que os objetivos principais do preparo do canal radicular são: a remoção de tecido pulpar infectado, material necrótico remanescente, dentina infectada, bem como a neutralização de bactérias e seus produtos, porém as diversidades anatômicas, como as encontradas nos canais achatados, podem vir a dificultar a limpeza do sistema de canais radiculares. Desta forma, diversos autores avaliaram a limpeza do canal com diferentes técnicas, por exemplo: ápico-cervical (step-back) ${ }^{8}$ força balanceada, ${ }^{9}$ técnicas ultrassônicas, ${ }^{10}$ bem como outras técnicas, concluindo que nenhuma é capaz de tocar em todas as paredes do canal e, por fim, acabam por deixar remanescentes que podem perpetuar a infecção endodôntica.

Já em 1968, Gutierrez e Garcia ${ }^{11}$ observaram áreas não tocadas pelos instrumentos em incisivos e caninos inferiores, relacionando com as áreas de prolongamentos vestibular e lingual destes canais ovalados, mesmo quando estes eram preparados com instrumentos de maior diâmetro.

Também realizando estudos com canais achatados, Rubin et al. ${ }^{12}$ observaram que após o preparo as paredes mesial e distal apresentaram-se sempre limpas, porém as paredes vestibular e lingual mostravam restos de tecido pulpar e pré-dentina, em virtude de não haver ação do instrumento nessas áreas.

$\mathrm{Na}$ tentativa de remover detritos localizados nas áreas vestibular e lingual, foi sugerido o uso do ultrassom na endodontia, dando margem a diversos estudos. ${ }^{10,13}$

Brosco et al. ${ }^{14}$ verificaram, em 1991, a capacidade de limpeza de canais radiculares de 20 incisivos inferiores sob luz do microscópio óptico. Os dentes foram preparados pela técnica biescalonada pura e associada ao ultrassom. Foram avaliados a $1 \mathrm{~mm}$ e $3 \mathrm{~mm}$ do ápice radicular. Os resultados mostraram que a associação da instrumentação manual com o ultrassom aumenta a capacidade de limpeza dos canais radiculares. Tal dado foi coincidente com os resultados de Holanda Pinto et al., ${ }^{15}$ que utilizaram avaliação em MEV, e a técnica de manual escalonada e ultrassônica com limas tipo K \#15, 20, 25.

Evidenciando as vantagens da associação da instrumentação dos canais radiculares com ultrassom, Torabinejad e Linda ${ }^{16}$ afirmam que 
esta associação proporciona canais mais limpos e com menor risco de transporte.

Siqueira Jr. et al. ${ }^{17}$ realizaram um estudo comparando a efetividade de cinco técnicas de instrumentação na limpeza do terço apical, utilizando como método de avaliação a histologia. Utilizaram 53 canais, divididos em cinco grupos - G1: técnica step back com limas K-Flexofile; G2: técnica step back com limas Ni-Ti manuais; G3: técnica ultrassônica com limas K-Flexofile; G4: Técnica da força balanceada; G5: técnica Canal Máster U. Os resultados demonstraram que quanto à limpeza os sistemas testados se equivalem, mas nenhuma técnica foi capaz de promover a limpeza total do sistema de canais radiculares, possivelmente pelas diversidades anatômicas internas do canal, deixando remanescentes de tecido em istmos e reentrâncias.

Em 2001, Wu et al. ${ }^{18}$ realizaram um estudo com 20 incisivos inferiores unirradiculares, a fim de observar recessos após o preparo. Os dentes foram preparados utilizandose a técnica da força balanceada, e foram posteriormente obturados, utilizando-se a técnica da condensação lateral a frio. Foram realizadas secções transversais a $5 \mathrm{~mm}$ e $3 \mathrm{~mm}$ do comprimento de trabalho, avaliadas à luz do estéreo-microscópio. Descreveram que a 5 $\mathrm{mm}$ do ápice radicular foram encontradas áreas não instrumentadas, havendo áreas preenchidas pelo material obturador (65\%), e áreas onde não houve tal preenchimento. Em $3 \mathrm{~mm}$ foram encontradas áreas com recessos preenchidas $(60 \%)$, e aquelas que não foram preenchidas no momento da obturação. Tais resultados demonstram a dificuldade de instrumentação e obturação de canais achatados.

Também utilizando a histologia como método de avaliação de remanescentes nas paredes do canal, Tan e Messer ${ }^{19}$ coletaram 30 molares com canais achatados, divididos em três grupos - G1: técnica ápice-coroa sem preparo cervical; G2: técnica ápice-coroa com preparo cervical prévio; G3: sistema LightSpeed. Para a avaliação histológica, os dentes foram preparados e seccionados a $5 \mu \mathrm{m}$ de espessura, em dois níveis apicais a $1 \mathrm{~mm} \mathrm{e} 3 \mathrm{~mm}$ do comprimento de trabalho. As secções transversais foram processadas e coradas por hematoxilina-eosina, levadas ao microscópio óptico sob aumento de 63X para avaliação da limpeza, sendo realizadas fotografias de cada corte. Sobre cada imagem, foi colocada uma grade dividindo a luz do canal em dez quadrantes, avaliados pelo sistema de scores adotado. Descreveram como resultados que cerca de $80 \%$ do canal mostrouse limpo quando instrumentado pelo sistema LightSpeed; por outro lado, a instrumentação manual sem o preparo cervical prévio se mostrou menos eficaz.

Sasaki etal. ${ }^{20}$ avaliaram a limpeza das paredes do canal radicular em 18 incisivos inferiores após preparo com o sistema Protaper. Ao final do preparo, a porção apical de cada dente foi seccionada e então processada histologicamente, e corada por hematoxilina-eosina. As amostras foram cortadas transversalmente a $6 \mu \mathrm{m}$ de espessura. As lâminas foram levadas ao microscópio óptico à magnitude de 40X. Os resultados demonstraram que cerca de 6,49\% da área do canal apresentou locais que não sofreram a ação dos instrumentos rotatórios, demonstrando que estes instrumentos não são capazes de tocar em todas as paredes de canais com achatamento mésio-distal. ${ }^{21}$

No mesmo ano, Huang et al. ${ }^{22}$ coletaram 20 incisivos permanentes humanos e avaliaram sua configuração anatômica. Para tal, os dentes foram incluídos em resina, cortados transversalmente no nível cervical, médio e apical. As secções foram analisadas em estéreomicroscópio quanto ao: número de canais, forma e presença de istmo. Os resultados demonstraram que para o terço apical foram encontrados $44,5 \%$ de canais achatados, $48,7 \%$ de canais com istmos, $15 \%$ de canais com dois canais. Concluíram que a morfologia do canal radicular em incisivos inferiores é bastante variada, mas achados como canais ovais e a presença de istmo são comuns, o que pode dificultar a terapia endodôntica.

Em estudo que avaliou a limpeza em canais achatados por meio de secções transversais, Grande et al. ${ }^{23}$ utilizaram a técnica AET (Anatomic Endodontic Therapy) e o sistema Protaper no preparo de 50 pré-molares inferiores com canais achatados. Os autores concluíram que nos terços cervical e médio a técnica AET foi mais efetiva na modelagem do canal, sendo que no terço apical os sistemas comportaram-se de maneira semelhante, sendo mais uniformes.

De um modo geral, a literatura demonstra que a instrumentação rotatória com instrumentos em Ni-Ti tem ação limitada, deixando em canais achatados áreas localizadas vestíbulo-lingualmente não preparadas, e provavelmente infectadas. ${ }^{17,18,20}$ 
Diante do exposto, o presente trabalho teve por objetivo analisar a morfologia apical de incisivos inferiores extraídos em diferentes secções transversais por meio de lupa estéreomicroscópica, com o objetivo de conhecer a morfologia e a configuração do canal radicular deste grupo de dentes, para que desta forma se possa nortear o profissional na correta limpeza e modelagem do sistema de canais radiculares.

\section{Material e Métodos}

Foram coletados 100 incisivos centrais e laterais inferiores de indivíduos entre 20 e 30 anos, recém-extraídos, com rizogênese completa e comprimento médio de $18 \mathrm{~mm}$. Os dentes foram adquiridos nas Clínicas Odontológicas da Universidade Federal do Pará (UFPA) e em ambulatórios do Sistema Único de Saúde (SUS). Para cada amostra, o doador assinou um termo de consentimento livre e esclarecido, autorizando a utilização do dente no trabalho, conforme documento aprovado pelo Comitê de Ética e Pesquisa em Seres Humanos do Centro de Ciências da Saúde da UFPA (protocolo 152/07 CEP-CCS/UFPA). Os dentes foram limpos com curetas periodontais e ultrassom, numerados e armazenados em água destilada sob refrigeração.
Para a análise da morfologia apical do canal radicular foram realizadas secções transversais a 1,3 e $5 \mathrm{~mm}$ do ápice radicular pelo uso de disco de aço diamantado dupla face $N^{\circ}$ KG: 7012 (KG-Sorensen - São Paulo - Brasil), de 0,2 mm de espessura, acionado em baixa rotação.

Os cortes foram mergulhados em $\mathrm{NaOCl}$ a $1 \%$ para remoção de restos pulpares na luz e aderidos nas paredes do canal. Após isso, foram adaptados em lâminas de vidro com cola para isopor (ArtMaxi-Brasil), sendo identificados como A: $1 \mathrm{~mm}, \mathrm{~B}: 3 \mathrm{~mm}$, C: $5 \mathrm{~mm}$ aquém do ápice radicular.

Para análise, os cortes foram levados ao estéreo-microscópio Zeiss SV - M (Carl Zeiss Brasil Ltda.) em aumento de 50X, com câmera digital Canon (PowerShot A630) adaptada, sendo que para cada dente foram realizadas três imagens de acordo com o nível do corte $(1,3$, e $5 \mathrm{~mm}$ aquém do ápice radicular). Os critérios utilizados na avaliação foram: número de canais e forma (oval, oval longo, circular).

\section{Resultados}

$\mathrm{Na}$ avaliação da morfologia do canal radicular de incisivos inferiores, foram encontrados os resultados descritos na Tabela 1.

Tabela 1: PrevalÊNCIA DE NÚMERo E FORMA DE CANAIS NO TERÇO APICAL DE INCISIVOS CENTRAIS INFERIORES NOS DIFERENTES NÍVEIS ESTUDADOS

\begin{tabular}{cc|c|c|c|c}
\hline Nível & Circular & Oval & Oval longo & Dois Canais & Total de amostras \\
\hline A (1 $\mathbf{~ m m})$ & 16 & 36 & 48 & - & 100 \\
B (3 mm) & - & 36 & 64 & - & 100 \\
C (5 mm) & - & 28 & 64 & 8 & 100 \\
\hline
\end{tabular}

De modo geral, a maioria dos canais estudados apresentou-se com formato oval longo (Figura 1), atingindo $48 \%$ a $1 \mathrm{~mm}$, e $64 \%$ a $3 \mathrm{~mm}$ e $5 \mathrm{~mm}$, sendo seguido pelo formato oval (Figura 2) (cerca de 36\%). O formato circular foi encontrado apenas a $1 \mathrm{~mm}$ do ápice (16\%) (Figura 3). Observou-se, nos dentes estudados, que a frequência de dois canais é baixa, perfazendo um total de $8 \%$ (Figura 4).

\section{Discussão}

O sucesso da terapia endodôntica depende, entre outros fatores, da habilidade do profissional em encontrar, limpar, modelar

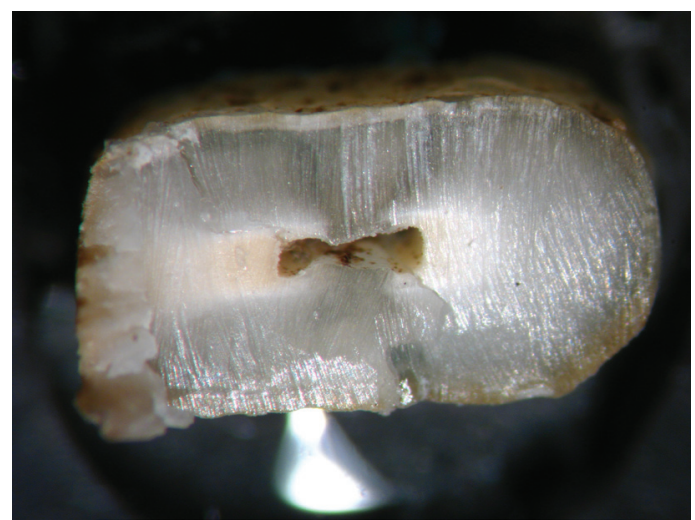

Figura 1: SeCÇão tranSVERSAL do CANAL RADicUlar COM FORMATO OVAL-LONGO. 


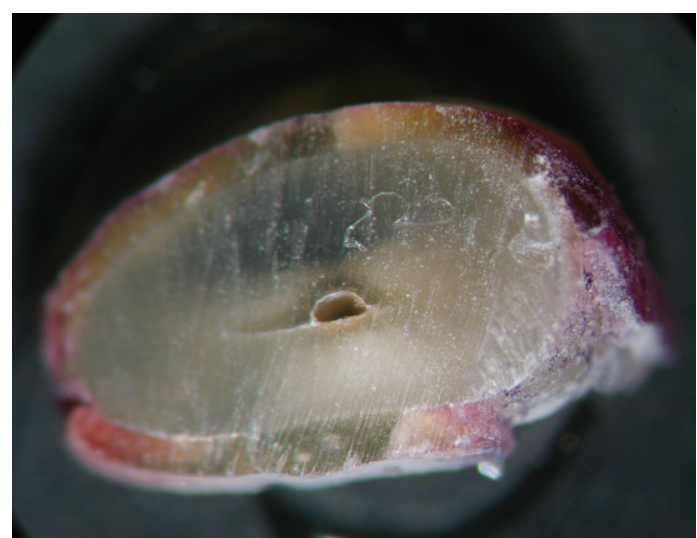

Figura 2: Seç̧ão transversal do canal radicular COM FORMATO OVAL.

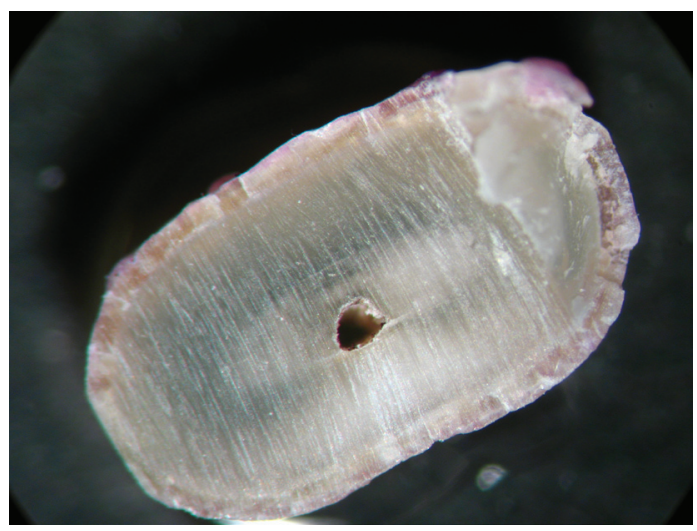

Figura 3: SECÇÃo transVERSAL DO CANAL RADICULAR COM FORMATO CIRCULAR.

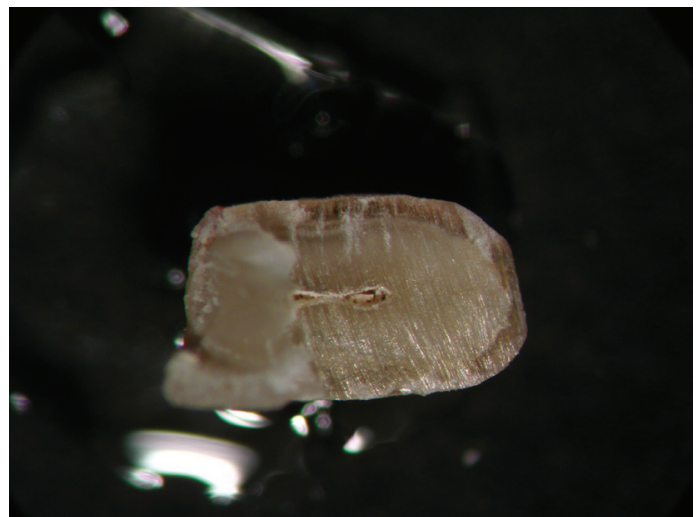

Figura 4: Secção transversal do CANAL RADicular EVIDENCIANDO O SEGUNDO CANAL.

e selar corretamente o sistema de canais radiculares, porém as diversidades anatômicas, como as encontradas em canais achatados, podem vir a dificultar a terapia, levando muitas vezes ao fracasso.

No presente estudo, a ocorrência de dois canais distintos em cada nível apical foi rara, cerca de $8 \%$, corroborando os achados de Vertucci, ${ }^{4}$
Mauger et al., ${ }^{2}$ Uma et al., ${ }^{7}$ sendo no presente estudo encontrado somente a $5 \mathrm{~mm}$ do ápice radicular, dados diferentes dos encontrados por Kartal e Yanikoglu, ${ }^{24}$ que descreveram que $45 \%$ dos dentes estudados apresentaram dois canais, quando se considerou o mesmo nível apical.

O formato circular foi encontrado apenas a $1 \mathrm{~mm}$ do ápice, perfazendo cerca de $16 \%$ dos canais avaliados, corroborando dados da literatura, que relata a tendência de canais circulares à medida que se avança em direção apical. $2,3,18$

O formato oval encontrado nos canais estudados foi de $36 \%$, semelhante aos dados encontrados por Mauger et al., ${ }^{2}$ que demonstraram a presença de canais achatados em $42 \%$ dos dentes estudados.

A incidência de canais com o formato oval longo no presente estudo foi alta, sendo que para cortes a $1 \mathrm{~mm}$ do ápice a incidência foi de $48 \%$, e em $3 \mathrm{~mm}$ e $5 \mathrm{~mm}$, de $64 \%$, dados semelhantes aos encontrados na literatura., ${ }^{2,3,22}$

Em 1956, Grossman ${ }^{25}$ afirmou que o preparo biomecânico é o método mais eficaz de limpeza, retificação e alisamento das superfícies dos canais radiculares, ressaltando que a dilatação mínima para qualquer tipo de canal deve corresponder ao diâmetro \#30. Por outro lado, na tentativa de preparar a porção apical de canais achatados com a utilização de instrumentos de maior calibre, o profissional pode causar perfurações laterais, em virtude do acentuado achatamento mésio-distal encontrado em incisivos inferiores. ${ }^{2}$

Ao final do preparo do canal, podese realizar a irrigação final com hipoclorito de sódio, com uma lima de fino calibre introduzida a $2 \mathrm{~mm}$ do comprimento de trabalho no interior do canal radicular energizada pelo ultrassom pelo período de três minutos. A energização promove circulação do líquido irrigador ao redor da lima ativada, além do efeito cavitacional, e assim promove limpeza das paredes do canal. ${ }^{26}$

De modo geral, a literatura demonstra que ainda não existe uma técnica que prepare em sua totalidade canais com achatamento mésio-distal. Os preparos circulares promovidos pela instrumentação seja rotatória ou manual, não abrangem todas as paredes do canal radicular, pois promovem preparo circular correspondente à secção transversal do instrumento, o que deixa a limpeza do material remanescente nas paredes do canal a cargo das soluções químicas auxiliares, o que pode levar ao insucesso da terapia. ${ }^{2,18,21}$ 


\section{Conclusão}

Diante dos resultados obtidos pela aplicação da metodologia apresentada, é pertinente afirmar:

1. A incidência de canais ovais alongados é grande no terço apical, e os preparos circulares promovidos pela instrumentação podem não abranger todas as paredes do canal radicular, deixando áreas sem serem tocadas, com restos de material infectado, ficando a limpeza a cargo das substâncias químicas auxiliares, o que pode levar ao insucesso da terapia.

2. No presente estudo foi observado que a ocorrência de canais do tipo oval longo é grande, perfazendo um total de $48 \%$ no nível mais apical estudado $(1 \mathrm{~mm})$, e ainda que este achatamento permanece à medida que se caminha em direção coronal.

3. Há necessidade de criação de protocolos para o preparo de canais achatados, uma vez que a ocorrência deste tipo de canal é grande, e a literatura demonstra que ainda não há uma técnica capaz de promover a completa limpeza de todas as paredes.

\section{REFERÊNCIAS BibLIOGRÁFICAS}

1. Green D. Double canals in single roots. Oral Surg Oral Med Oral Pathol. 1973 May; 35(5):689-96.

2. Mauger MJ, Schindler WG, Walker WA. An evaluation of canal morphology at different levels of root resection in mandibular incisors. J Endod. 1998; 24: 607-9.

3. Wu MK, R'oris A, Barkis D, Wesselink PR. Prevalence and extent of long oval canals in the apical third. Oral Surg Oral Med Oral Pathol Oral Radiol Endod. 2000 Jun; 89(6):739-43.

4. Vertucci FJ. Root canal anatomy of the mandibular anterior teeth. J Am Dent Assoc 1974; 89:369-71.

5. Miyashita M, Kasahara E, Yasuda E, Yamamoto A, Sekizawa T. Root canal system of the mandibular incisor. J Endod. 1997 Aug; 23(8):479-84.

6. Al-Qudah AA, Awawdeh LA. Root canal morphology of mandibular incisors in a Jordanian population. Int Endod J. 2006; 39:873-7.

7. Uma $\mathrm{CH}$, Ramachandran $\mathrm{S}$, Indira $\mathrm{R}$, Shankar P. Canal and isthmus morphology in mandibular incisors: an in vitro study. Endodontol, 2004; 1(6): 7-11.
8. Clem, W.H. Endodontics: the adolescent patient. Dent. Clin. North Am. 1969; 13: 483-93.

9. Roane JB, Sabala CL, Duncanson MG Jr. The "balanced force" concept for instrumentation of curved canals. J Endod. 1985 May; 11(5):203-11.

10. Martin H. Ultrasonic disinfection of the root canal. Oral Surg Oral Med Oral Pathol $1976 \mathrm{Jul} ; 42(1): 92-9$.

11. Gutiérrez, JH; García, J. Microscopic and macroscopic investigation on results of mechanical preparation of root canals. Oral Surg. 1968 Jan; 25(1):108-16.

12. Rubin $\mathrm{L}$, et al. The effect of instrumentation and flushing ofreshly extracted teeth in endodontic therapy: a scanning electron microscope study. J Endod. 1979 Nov; 5 (11):328-35.

13. Richman, MJ. The use of ultrasonics in root canal therapy and root resection. J Dent Med. 1957 Jan; 12(1):12-8.

14. Brosco HB, et al. Análise comparativa do preparo biomecânico biescalonado isolado ou coadjuvado por ultra-sonificação na limpeza dos canais radiculares. Rev Bras Odontol. 1991 Nov/Dez; 48(6):21-5.

15. Holanda Pinto SA, Bramante CM, Berbert A. Avaliação da limpeza de canais radiculares obtida pelas técnicas de instrumentação manual, ultra-sônica e combinação de ambas. Rev Bras Odontol. 1991 Mai/Jun; 48(3):2-12.

16. Torabinejad, M, Linda, L. Passive stepback technique. Oral Surg. 1994 Apr; 77(4):402-5.

17. Siqueira JF Jr, Araujo MC, Garcia PF, Fraga RC, Dantas CJ. Histological evaluation of the effectiveness of five instrumentation techniques for cleaning the apical third of root canals. J Endod. 1997 Aug; 23(8):499502.

18. Wu MK, Wesselink PR. A primary observation on the preparation and obturation of oval canals. Int Endod J. 2001 Mar; 34(2):137-41.

19. Tan BT, Messer HH. The quality of apical canal preparation using hand and rotary instruments with specific criteria for enlargement based on initial apical file size. J Endod. 2002 Sep; 28(9):658-64.

20. Sasaki EW, Versiani MA, Perez DE, SousaNeto MD, Silva-Sousa YT, Silva RG. Ex vivo analysis of the debris remaining in 
flattened root canals of vital and nonvital teeth after biomechanical preparation with Ni-Ti rotary instruments. Braz Dent J. 2006; 17(3):233-6.

21. Fariniuk LF, Baratto-Filho F, da CruzFilho AM, de Sousa-Neto MD. Histologic analysis of the cleaning capacity of mechanical endodontic instruments activated by the ENDOflash system. J Endod. 2003 Oct; 29(10):651-3.

22. Huang DM, Hao YQ, Luo SG, Jia LH, Zhou XD. Morphology of cross-sectioned root canal in Chinese mandibular permanent incisors. Sichuan Da Xue Xue Bao Yi Xue Ban. 2006 Nov; 37(6):916-8.

23. Grande NM, Plotino G, Butti A, Messina F, Pameijer CH, Somma F. Crosssectional analysis of root canals prepared with NiTi rotary instruments and stainless steel reciprocating files. Oral Surg Oral Med Oral Pathol Oral Radiol Endod. 2007 Jan; 103(1):120-6.

24. Kartal N, Yanikoğlu FC. Root canal morphology of mandibular incisors. J Endod. 1992 Nov; 18(11):562-4.

25. Grossman, L.I. Endodontics practice. 7. ed. Philadelphia: Lea \& Febiger; 1978.

26. Jensen SA, Walker TL, Hutter JW, Nicoll BK. Comparison of the cleaning efficacy of passive sonic activation and passive ultrasonic activation after hand instrumentation in molar root canals. J Endod. 1999 Nov; 25(11):735-8.

Recebimento: 29/5/2009 Aprovação: 11/4/2011 